TITLE:

\title{
Applicability condition of time-temperature superposition principle (TTSP) to a multi-phase system
}

$\operatorname{AUTHOR}(\mathrm{S})$ :

Nakano, Takato

\section{CITATION:}

Nakano, Takato. Applicability condition of time-temperature superposition principle (TTSP) to a multi-phase system. Mechanics of Time-Dependent Materials 2013, 17(3): 439-447

ISSUE DATE:

2013-08

URL:

http://hdl.handle.net/2433/192984

\section{RIGHT:}

The final publication is available at Springer via http://dx.doi.org/10.1007/s11043-0129195-8.; This is not the published version. Please cite only the published version.; この論 文は出版社版でありません。引用の際には出版社版をご確認ご利用ください。 
1 Title: Applicability condition of time-temperature superposition principle (TTSP) to a multi-phase 2 system

3

4 Author: Takato Nakano

5

6 Affiliation: Laboratory of Biomaterials Design, Division of Forest and Biomaterials Science, Graduate

7 School of Agriculture, Kyoto University

8

9 address: Kita-shirakawa, Kyoto, 606-8502 Japan.

10

11 e-mail: tnakano@kais.kyoto-u.ac.jp

12

13 phone: +81-75-753-6234

14

15

16

17

18 
19

20

21

\section{Abstract}

The applicability condition of the time-temperature superposition principle (TTSP) to a multi-phase system is analytically discussed assuming a mixture law. It was concluded that the TTSP does not hold for a multi-phase system in general but does hold for a multi-component system in which some components have the same temperature dependence and the others have no temperature dependence. On the basis of the results, the application of the TTSP to plant materials such as wood and bamboo was examined using a mixture law and a stretched-exponential function having a characteristic relaxation time $\tau_{0}$ and a stretching parameter $\beta$. Wood can be treated as a multi-phase system consisting of a framework (f) and matrix (m). In this case, it was expected that the TTSP holds for the matrix in the shorter time region $t<<\tau_{0 f}$ under $T<T_{g f}$, while the TTSP holds for the framework in the longer time region $t>>\tau_{0 m}$ under $T>T_{g m}$, where $t$ and $T_{g}$ is a measurement time and the glass transition temperature, respectively

Keywords: time-temperature superposition principle, relaxation, multi-phase, wood 


\section{Introduction}

In this work, the applicability of the time-temperature superposition principle (TTSP) to a multi-phase system is analytically discussed. The TTSP is applicable only under limited conditions (Fesko and Tschoegl, 1971; Nakano, 1995; Tschoegl et al., 2002). According to Tschoegl (2002), the TTSP definitely does not apply to multi-phase materials such as graft copolymers, hybrid materials, and some other systems. In this study, we analytically identified both applicable and non-applicable conditions and applied the TTSP to plant materials considered as multi-phase systems.

The TTSP has been widely applied. However, most of the studies have not strictly considered thermorheological simplicity. In order for the TTSP to be applicable, two requirements must be satisfied: curves measured at different temperatures can be superimposed, and all system responses are equally dependent on temperature. It should be noted that just a smooth curve established by superimposing curves at different temperatures does not validate the TTSP.

Fesko and Tschoegl (1971) found that the TTSP does not hold for multi-phase systems and that the shift factor is time-dependent. Kaplan and Tschoegl (1974) applied the TTSP to two-phase polyblends and concluded that the master curve could not be constructed using just one shift factor. Tschoegl et al. (1999) reviewed the effect of temperature and pressure on the mechanical properties of polymeric materials and again pointed out limitations of the TTSP. Nakano (1995) also reported non-applicability of the TTSP to wood when viewed as a multi-phase system. Nevertheless, rigorous application of the TTSP for multi-phase systems is highly desirable because it would enable the prediction of relaxation properties over longer and shorter time intervals and at lower and higher frequencies.

The relaxation behavior of wood has been examined extensively. Samarasinghe et al. (1994) reported that the relaxation curve obtained over a long time interval agreed with a master curve derived from curves at various temperatures. Bond et al. (1997) reported that the TTSP holds for creep in compression and tension for yellow poplar, Douglas fir, and southern pine. Irvin (1980) and Salmén (1984) reported that the Williams-Landel-Ferry (WLF) equation held for isolated lignin and in situ lignin, respectively. Stephen et al. (1987) claimed that the TTSP should be restricted to the amorphous components of wood. Dlouhá et al. (2009) examined five wood species and did not definitively conclude that the TTSP was applicable. In an analytical study, Nakano (1995) indicated that the TTSP was not applicable for wood as a multi-phase system. The TTSP has also been applied to various multi-phase systems (e.g., Simon and Ploehin, 1999; Brostow and D’Souza, 1999; Akinay et al., 2002; Macaúbas and Demarquette, 2002; Barbero and Julius, 2003; Tajvidi et al., 2005; Liu et al., 2009; Barbero and Ford, 2004; Nakada et al., 2011).

The application of the TTSP requires that all relaxation processes depend equally on 
temperature. This requirement has not been rigorously considered in the literature. Thus, the validity of the TTSP must be strictly considered before applying it to a multi-phase system. In some cases, authors have pointed out the non-applicability of the TTSP to multi-phase systems but did not suggest conditions that might make it applicable.

In this work, the applicability of the TTSP to a multi-phase system has been formulated using a mixture law. The analytical conditions for applicability are discussed. The stretched-exponential function has been applied to analysis of wood as a multi-phase system.

\section{Results and discussion}

\section{Time-temperature superposition principle (TTSP)}

The TTSP implies that time or frequency is equivalent to temperature. That is, time or frequency at temperature $T_{1}$ is related to that at temperature $T_{0}$ by the following equations:

$$
t\left(T_{1}\right)=\frac{t\left(T_{0}\right)}{a_{T}},
$$

$$
\omega\left(T_{1}\right)=\omega\left(T_{0}\right) a_{T},
$$

where $t\left(T_{0}\right)$ and $t\left(T_{1}\right)$ are times and $\omega\left(T_{1}\right)$ and $\omega\left(T_{0}\right)$ are frequencies at temperatures $T_{0}$ and $T_{1}$. These relationships make it possible to establish a curve ("master curve") as a function of the logarithm of the time or frequency by superimposing the curves measured at various temperatures. Rouse theory predicts the TTSP and indicates that it should be applied to the glass transition and rubbery regions where relaxation processes relate to the free volume. Application of the TTSP to polymers in the glassy state and to crystalline polymers has been reported (Faucher, 1959; Nagamatsu and Yoshitomi, 1959; Yoshitomi et al., 1958). The authors reported different mechanisms having different temperature dependencies. It should be stressed that equations (1) and (2) imply that all relaxation processes depend equally upon temperature.

Time at temperature $T_{1}, t\left(T_{1}\right)$, is reduced to that at temperature $T_{0}, t\left(T_{0}\right)$, using equations (1) and (2). Figure 1 shows how a relaxation curve measured at temperature $T_{1}$ can be superimposed on one measured at temperature $T_{0}$ using a small vertical shift.

Using equations (1) and (2), we have

$$
E\left(t / a_{T}, T_{0}\right)=\frac{T_{0} \rho_{0}}{T \rho} E\left(t, T_{1}\right),
$$




$$
E^{\prime \prime}\left(\omega a_{T}, T_{0}\right)=\frac{T_{0} \rho 0}{T \rho} E^{\prime \prime}\left(\omega, T_{1}\right),
$$

where $E(t, T), E^{\prime}(\omega, T)$, and $E^{\prime \prime}(\omega, T)$ are the relaxation modulus, storage modulus, and loss modulus at temperature $T$. The factor $T_{0} \rho_{0} / T \rho$ is related to thermal expansion and is almost unity. Equations (3) to (5) show that two curves can be superimposed on a logarithmic scale by shifting on the basis of $\ln a_{T}$. Similar equations can also be derived for viscosity, static compliance, and dynamic compliance.

Equations (1) and (2) require that all relaxation processes depend equally on temperature. A smooth curve established by superimposing curves at different temperatures does not necessarily validate the TTSP and is rheologically nonsensical if the above requirement does not hold. The TTSP implies equivalence between time and temperature but not superimposition of curves simply by shifting.

The applicability of the TTSP to multi-phase systems for stress relaxation processes is discussed below. A thermal correction factor $T_{0} \rho_{0} / T \rho=1$ is used to simplify the analysis. The same analysis should be valid for dynamic behavior.

\section{Applicability of the TTSP to a multi-phase system}

Fesco and Tschoegl (1971) theoretically examined the application of the TTSP to a thermorheologically complex system and found that the shift factor was a function of both temperature and time. Subsequently, Kaplan and Tschoegl (1974) derived the master curve for a two-phase system using the master curve of each component and Takayanagi's mixture law. They estimated the time dependence of the shift factor using the distance between the obtained master curve of each component and an experimental curve. Their results showed that the master curve cannot be established from relaxation curves at various temperatures simply by shifting on a logarithmic scale. Here, we analytically determine the conditions of applicability of the TTSP for a multi-phase system by extending the work of Kaplan and Tschoegl (1974).

Assuming a mixture law that is independent of temperature, a situation that holds for most multi-phase polymers, the relaxation modulus at temperature $T$ of a multi-phase system is represented by

$$
E^{n}(t, T)=\sum_{i} \theta_{i} E_{i}^{n}(t, T)
$$

where $\theta_{i}$ and $E_{i}$ are the volume fraction and the relaxation modulus for component $i$, respectively, and $n$ is the mixture state parameter and $-1 \leq n \leq 1$. 
Assuming that the TTSP is valid for each component $i$, the relationship between moduli at different temperatures $T_{0}$ and $T_{1}$ is described by equation (7):

$$
\text { - } E_{i}\left(t, T_{1}\right)=E_{i}\left(t / a_{T_{i}}, T_{0}\right) . \quad\left(T_{0} \rho_{0} / T \rho=1\right),
$$

If the temperature dependence of all components is equal, that is $a_{T 0}=a_{T 1}=\cdots=a_{T i}=\cdots \equiv a_{T}$, then the modulus of the entire system is:

$$
E^{n}\left(t, T_{1}\right)=\sum_{i} \theta_{i} E_{i}^{n}\left(t, T_{1}\right)=\sum_{i} \theta_{i} E_{i}^{n}\left(t / a_{T}, T_{0}\right)
$$

This equation is equal to $E^{n}\left(t / a_{T}, T_{0}\right)$.

The above result means that the TTSP holds for a multi-phase system when the TTSP is valid for all components and more they have the same shift factor. This is the simplest case that can be used to validate the TTSP. Our interest is the applicability of the TTSP to a multi-phase system when the temperature dependence of at least one component differs from the others, even though the TTSP is valid for each component.

In such a case, when the component that differs from the others is represented by subscript $p$, equation (8) can be rewritten as

$$
E^{n}\left(t, T_{1}\right)=\theta_{p} E_{p}^{n}\left(t / a_{T p}, T_{0}\right)+\sum_{i \neq p} \theta_{i} E_{i}^{n}\left(t / a_{T}, T_{0}\right),
$$

where $a_{T p}$ and $a_{T}$ are the shift factors of the different component and the others, respectively. Equation (9) means that a curve for $T_{1}$ does not superimpose on that for $T_{0}$ by the same shift factor because the shift factors $a_{T p}$ and $a_{T}$ differ in the first term and the second term on the right-hand side in the equation. This argument is equally valid when more than two components differ from each other and more have shift factors that differ from the others.

Equation (9) indicates that curves at different temperatures cannot be superimposed using a common shift factor. However, they can be superimposed using the procedure of Kaplan and Tschoegl (1974). That is, we first establish a master curve for each component using a shift factor for the pure component and then calculate the master curve of the entire system using a mixture law. This procedure is acceptable only when the volume fraction, relaxation properties, and shift factor for each component are known, and the interaction between the components is negligible. That is, equation (9) indicates that superimposition using a single shift factor is impossible for a multi-phase system. The above discussion implies that the TTSP is not valid for a multi-phase system containing components having different temperature dependencies.

To date, the applicability of TTSP has been discussed for a multi-phase system in which the components had different temperature dependencies, although the TTSP is not valid for a multi-phase system containing components having different temperature dependencies (Fesco and Tschoegl, 1971; Kaplan and Tschoegl, 1974; Nakano, 1995). Some studies have shown that the TTSP holds completely 
or partially for some multi-phase systems, for example polymer liquid crystal and natural products such as wood, respectively. This fact appears to suggest a restrictive condition for applicability to a multi-phase system of TTSP. Thus, we aimed to identify the condition for which the TTSP is valid. We examined a multi-phase system in which some components had slight or no temperature dependence.

When the $0^{\text {th }}$ to $q^{\text {th }}$ components do not have temperature dependencies, relaxation processes are frozen or completed, and the other components have equal temperature dependencies, the relaxation modulus at $T_{1}$ is given by

$$
E^{n}\left(t, T_{1}\right)=\sum_{i=0}^{q} \theta_{i} E_{i}^{n}\left(t, T_{1}\right)+\sum_{i>q} \theta_{i} E_{i}^{n}\left(t, T_{1}\right) .
$$

Here, because the $0^{\text {th }}$ to $\mathrm{q}^{\text {th }}$ components do not have temperature dependencies, the first term of the right-hand side in equation (10) is constant. Thus, equation (10) reduces to

$$
E^{n}\left(t, T_{1}\right)=\text { const. }+\sum_{i>q} \theta_{i} E_{i}^{n}\left(t, T_{1}\right) .
$$

As the other components have equal temperature dependencies,

$$
E^{n}\left(t, T_{1}\right)=\text { const. }+\sum_{i>q} \theta_{i} E_{i}^{n}\left(t / a_{T}, T_{0}\right)
$$

This is equal to $E^{n}\left(t / a_{T}, T_{0}\right)$. That is, the TTSP is valid.

The above discussion shows that, in general, the TTSP holds for a multi-phase system when the TTSP is valid for all components and their shift factors are identical. The TTSP is not valid for a system that contains at least one component having different temperature dependence. Moreover, the TTSP holds for a multi-phase system in which some of the components have no or nearly no temperature dependencies and the others have the same temperature dependence.

\section{Application of TTSP to wood as a multi-phase system}

Wood has a complex high-order structure and consists of multiple components, e.g., cellulose, hemicellulose, and lignin. The cell wall of wood contains these three components. Cellulose is a rigid polymer and is the primary component. It is semi-crystalline and constitutes the framework of the cell wall; the cellulose is embedded in a matrix consisting of hemicellulose and lignin. That is, wood is made up of both a framework and matrix having longer and shorter relaxation times. The relaxation process of a multi-phase system with such components thus consists of two processes. Wood and bamboo have characteristic relaxation behaviors, e.g., "mechano-sorptive behaviors" which is the peculiar relaxation behavior under changing moisture content and whose mechanism has not elucidated (Armstrong and Christensen, 1961; Gibson, 1965; Grossman, 1976). The relaxation 
properties of wood have been examined extensively, and those of bamboo recently, via microstructural analysis (Aoyagi and Nakano, 2009; Tsubaki and Nakano, 2010; Kanzawa et al., 2011).

Wood can be regarded as a multi-phase system consisting of a framework (subscript $f$ ) and a matrix (subscript $m$ ). The temperature dependencies of their relaxation properties are expected to be quite different, that is, $a_{T m} \neq a_{T f}$. In general, the TTSP does not hold for wood, according to the above discussion. However, Irvin (1980), Salmén (1984), Stephen et al. (1987), and Laborie (2004) reported that lignin, the main component of the matrix, obeys the WLF equation. The shift factor of the framework is expected to obey the Arrhenius equation because of local contributions to the relaxation of microfibrils (Ferry, 1980). In the following discussion, we examine the applicability of the TTSP to wet wood because in this state, the relaxation properties of a framework and matrix are expected to be very different.

Wood is porous biomaterial which consists of vacant space and wood cell substance. Thus, the high-order structure will affect Young's modulus. Its contribution is represented by the front factor in the following equation obtained by using a mixture low (Ohgama and Yamada、1974; Gibson and Ashby, 1997; Nakao and Nakano, 2011):

$$
E(t)=\Phi(\theta, \xi) E_{S}(t),
$$

where $\Phi(\theta, \xi)$ is the front factor related to the shape factor $\xi$ and the volume fraction of wood substance $\theta$, and $E_{S}$ is Young's modulus of the wood substance. Below $100^{\circ} \mathrm{C}, \Phi(\theta, m)$ is almost independent of time and temperature. Accordingly, $E(t)$ reduces to $E_{S}(t)$.

Reports have stated that the TTSP holds totally or partially for wood, although the above discussion stated that the TTSP generally does not hold for a multi-phase system. We resolve this discrepancy below.

In the following discussion, Young's moduli of the wood components are represented by a stretched-exponential function having characteristic relaxation time $\tau_{0}$ and stretching parameter $\beta$. This function is appropriate because relaxation properties are characterized by two parameters. This approach has been applied to the analysis of the effects of water adsorption on the stress relaxation of wood (Nakao and Nakano, 2011). Equivalence between a stretched-exponential function and the formulation by the linear viscoelastic theory has been clarified by Lindsey and Patterson (1987).

Now, consider wet wood as a two-phase system consisting of a framework and a matrix: the former is cellulose and the latter is hemicellulose plus lignin. Using the stretched-exponential function, Young's moduli of the framework and the matrix are represented by

$$
E_{m}(t)=E_{m}(0) \exp \left[-\left(\frac{t}{\tau_{0 m}}\right)^{\beta_{m}}\right]+E_{m}(\infty),
$$

$$
E_{f}(t)=E_{f}(0) \exp \left[-\left(\frac{t}{\tau_{0 f}}\right){ }^{\beta}{ }_{f}\right]+E_{f}(\infty)
$$


251

252

253

254

255

256

257

258

259

260

261

262

263

264

265

266

267

268

269

270

271

272

273

274

275

276

277

278

279

280

281

282

283

284

285

where subscripts $f$ and $m$ are the framework and the matrix, respectively, $E_{i}(0)$ and $E_{i}(\infty)$ $(i=f, m)$ are the instantaneous Young's modulus and the long-time asymptote (equivalent Young's modulus), respectively. Then, according a mixture law, Young's modulus of wood is represented by

$$
E_{S}{ }^{n}(t)=\theta_{m} E_{m}^{n}(t)+\theta_{f} E_{f}^{n}(t) .
$$

Relaxation properties of both the framework and matrix are expected to differ remarkably under the wet condition. This is because the glass transition temperatures of wet wood components are much more than $100^{\circ} \mathrm{C}$, less than room temperature, and about $60^{\circ} \mathrm{C}$ for cellulose, hemicellulose, and lignin, respectively (Goring, 1963; Irvine, 1980, 1984; Salmén, 1982, 1998), so that $T_{g f}$ and $T_{g m}$ differ from each other.

The above discussion concluded that the TTSP holds for a multi-phase system when one phase obeys the relaxation process while the others scarcely contribute to the relaxation. Define category (i) as the condition under which the matrix obeys the relaxation process while the framework scarcely contributes to the relaxation and category (ii) as the opposite condition. For our system,

(i) $T<T_{g f}$ and $t<<\tau_{0 f}$

(ii) $T>T_{g m}$ and $t>\tau_{0 m}$

Previous work (Irvin, 1980; Salmén, 1984; Stephen et al., 1987) indicated that the TTSP is valid for lignin in the matrix of the cell wall. The matrix consists of hemicellulose and lignin. There are approximately equal amounts of each, although the ratio differs somewhat between hardwoods and softwoods. As mentioned before, the glass transition temperature $T_{g}$ of hemicellulose is lower than those of the other components and well below room temperature when wet. On the other hand, the $T_{g}$ of lignin is about $60^{\circ} \mathrm{C}$ even when wet. Thus, lignin is the phase that obeys the relaxation process of the matrix from room temperature to $100^{\circ} \mathrm{C}$, while hemicellulose does not.

Now, we consider the condition $t<<\tau_{0 f}$. Considering $t / \tau_{0 f} \approx 0$ in the exponential term in equation (11), equation (11) becomes

$$
E_{f}(t) \approx E_{f}(0)+E_{f}(\infty)=\text { const. } \equiv K_{f}
$$

Thus, equation (15) reduces to

$$
E_{S}^{n}(t)=\theta_{m} E_{m}^{n}(t)+\theta_{f} K_{f}^{n} .
$$

Equation (17) shows that the matrix obeys the relaxation process for wood and the framework does not contribute. The relaxation modulus measured at two temperatures $T_{0}$ and $T_{1}$ under $T_{0}<T_{1}<<T_{g f}$ is represented by

$$
\begin{aligned}
& E_{S}{ }^{n}\left(t, T_{0}\right)=\theta_{m} E_{m}{ }^{n}\left(t, T_{0}\right)+\theta_{f} K_{f}{ }^{n} \\
& E_{S}{ }^{n}\left(t, T_{1}\right)=\theta_{m} E_{m}{ }^{n}\left(t, T_{1}\right)+\theta_{f} K_{f}{ }^{n}
\end{aligned}
$$

Now, considering $t\left(T_{1}\right)=t\left(T_{0}\right) / a_{T m}$ because TTSP is valid for the matrix,

$$
E_{m}\left(t, T_{1}\right)=E_{m}\left(t / a_{T m}, T_{0}\right) .
$$


Thus, equation (19) reduces to $E_{S}{ }^{n}\left(t, T_{1}\right)=\theta_{m} E_{m}{ }^{n}\left(t / a_{T m}, T_{0}\right)+\theta_{f} K_{f}{ }^{n}$.Accordingly,

$$
E_{S}{ }^{n}\left(t, T_{1}\right)=E_{S}{ }^{n}\left(t / a_{T m}, T_{0}\right)
$$

This equation implies that the TTSP is applicable for wood.

When the framework follows the relaxation process after the relaxation process of the matrix is complete, that is, under the condition $t \gg \tau_{0 m}$,

$$
E_{m}(t) \approx E_{m}(\infty)=\text { const } \equiv K_{m} .
$$

Thus,

Accordingly,

$$
E_{S}^{n}(t)=\theta_{m} K_{m}^{n}+\theta_{f} E_{f}^{n}(t)
$$

$$
E_{S}{ }^{n}\left(t, T_{1}\right)=E_{S}{ }^{n}\left(t / a_{T f}, T_{0}\right)
$$

Equation (24) also shows the validity of the TTSP.

It is clear from the above discussion that the TTSP holds for wood as a multi-phase system if condition (i) or (ii) is satisfied, in other words, in a limited temperature region or time period. The same discussion is also valid for dynamic behavior. Moreover, even if the TTSP is not valid for the framework, the TTSP is applicable for wood under the conditions $T_{0}<T_{1} \ll T_{g f}$ and $t<<\tau_{0 f}$. This is because wood consists of components having very different relaxation properties. This result supports reports stating that the TTSP is partially valid. It should be noted that the above discussion has not been applied to materials made of similar components.

\section{Conclusion}

The applicability of the TTSP to multi-phase systems was analytically discussed assuming a mixture law. It was concluded that the TTSP does not hold for multi-phase systems in general but does hold for the two-phase system when both components have the same temperature dependence and when both have no temperature dependence. The applicability of the TTSP to wet wood was examined using a mixture law and a stretched-exponential function having characteristic relaxation time $\tau_{0}$ and stretching parameter $\beta$. Wood can be considered as a multi-phase system made up of a framework (f) and a matrix $(\mathrm{m})$; at less than $100^{\circ} \mathrm{C}$, the framework (microfibrils) is rigid while the matrix (hemicelluloses and lignin) is flexible, because their glass transition temperatures $T_{g f}$ and $T_{g m}$ under wet condition are much more than $100^{\circ} \mathrm{C}$ and near or less than $60^{\circ} \mathrm{C}$, respectively. As a result, the TTSP holds for the matrix in the shorter time region $t<<\tau_{0 f}$ under $T<T_{g f}$, while the TTSP holds for the framework in the longer time region $t>\tau_{0 m}$ under $T>T_{g m}$. 


\section{References}

Aoyagi, S., Nakano, T. (2009) Effects of longitudinal and radial position on creep for bamboo (in Japanese), Zairyo (J. th Society of Mater. Sci. Jpn.), 58: 57-61.

Akinay A.E., Brostow, W., Castano, V.M., Maksimov, R., Olszynski, P. (2002) Time-temperature correspondence prediction of stress relaxation of polymeric materials from a minimum of data, Polym., 43; 3593-3600.

Armstrong, L.D., Christensen, G.N. (1961) Influence of moisture changes on deformation of wood under stress, Nature, No. 4791, August 26; 869-870.

Bueche, F. (1956) Deriviation of the WLF equation for the mobility of molecules in molten glasses, $J$ Chem. Phys., 24; 418-419.

Bond, B.H., Loferski, J., Tissaoui, J., Holzer S. (1997) Development of tension and compression creep models for wood using the time-temperature superposition principle, Forest Prod. J., 47; 97-103.

Brostow, W. and D’Souza, N.A. (1999) Creep and stress relaxation in alongitudinal polymer liquid crystal: prediction of the temperature shift factor, J. Chem. Phys., 110; 9706-9712.

Barbero, E.J. and Julius, M.J. (2004) Time-temperature-age viscoelastic behavior of commercial polymer blends and felt filled polymers, Mech. Adv. Mater Struc, 11; 287-300.

Barbero, E.J. and Ford, K.J. (2004) Equivalent time temperature model for physical aging and temperature effects on polymer creep and relaxation, J. Eng. Mater. Technol., 126; 413-419.

Ohgama, T. Yamada, T. (1974) Elastic modulus of porous materials (in Japanese), Mokuzai Gakkaishi, $20 ; 166-171$.

Dlouhá, J., Clair, B., Arnould, O.,Horácek, P., Gril J. (2009) On the time-temperature equivalency in green wood: Characterization of viscoelastic properties in longitudinal direction, Holzforschung, 63; 327-333

Faucher, J.A. (1959) Viscoelastic behavior of polyethelene and polypropylene, Trans. Soc. Rheology, 3; 81-93.

Ferry, J.D. (1980) "Viscoelastic Properties of Polymers" third ed., John Willey \& Sons, NewYork, pp.304-315.

Fesko, D.G. and Tschoegl, N.W. (1971) Time-temperature superposition in thermorheologically complex materials, J. Polym. Sci., 35; 51-69.

Gibson, L.J. and Ashby, M.F. (1997) "Cellular Solid" second edit., Cambridge University Press., Cambridge, pp.93-174.

Gibson, E.J. (1965) Creep of wood, Role of water and effect of a changing moisture content, Nature, No.4980, April 10; 213-215.

Goring, D.A.I. (1963) Thermal softening of lignin, hemicellulose and cellulose, Pulp and Paper 
Magazine of Canada, 64: T517-T527.

Grossman, P.U.A. (1976) Requirements of a model that exhibits mechano-sorptive behaviour, Wood Sci. Technol., 10,: 163-168.

Irvine, G. M. (1980) The glass transitions of lignin and its relevance to thermomechanical pulping, CSIRO Division of chemical technology research review, 33-43.

Irvine, G. M. (1984) The glass transitions of lignin and hemicellulose and their measurement by differential thermal, Tappi J., 67: 33-43.

Kanzawa, E.,Aoyagi, S., Nakano, T. (2011) Vascular bundle shape in cross-section and relaxation properties of Moso bamboo (Phyllostachys pubescens), Mater. Sci. \& Technol., C31; 1050-1054.

Kaplan, D. and Tschoegl N.W. (1974) Time-temperature superposition in two-phase polyblends, Polym. Eng. and Sci., 14; 43-49.

Kohlrausch, R. (1854) Theorie des Elektrischen Rückstandes in der Leidener Flasche, Pogg. Ann. Phys. CHem., 91; 179-214.

Liu, J. Cao, D.,Zhang, L., Wang, W. (2009) Time-temperature and time-concentration superposition of nanofilled elastmers: a molecular dynamics study, Macromol., 42, 2831-2842.

Laborie, Marie-Pierre. G., Salmén、Frazier, C.E. (2004). Cooperativity analysis of the in situ lignin glass transition, Holzforschung, 58; 129-133.

Lindsey, C.P., Patterson, G.D. (1980) Detailed comparison of the Williams-Watts and Cole-Davidson function. J. Chem. Phys. 73: 3348-3357.

Macaúbas, P.H.P. and Demarquette N.R. (2002) Time-temperature superposition principle applicability for blends formed of immiscible polymers, Polym. Eng. Sci., 42; 1509-1519.

Nakada, M, Miyano, Y., Cai, H., Kasamori, M.( 2011) Prediction of long-term viscoelastic behavior od amorphous resin beased on the time-temperature superposition principle, Mech. Time-Depend mater., DOI 10.1007/s11043-011-9139-8.

Nagamatsu, K. and Yoshitomi, T. (1959) On the viscoelastic properties of crystalline polymers: II Polytrifuluorochoroethylene, J. Colloid Sc, 14; 377-384.

Nakao, S. and Nakano, T. (2011) Analysis of molecular dynamics of moist wood components by applying the stretched exponential function, J. Mater. Sci., 46; 4748-4758.

Nakano, T. (1995) Time-temperature superposition principle on relaxation behavior of wood as a multi-phase material, Holz als Roh- und Werkstoff, 53; 39-42.

Back, E.L. and Salmén, L. (1982) Glass transitions of wood components hold implications for molding and pulping processes, Tappi, 65; 107-110.

Salmén, L. (1984) Viscoelastic properties of in situ lignin under water-satrated conditions, J. Mater. Sci., 19; 3090-3096.

Salmén, L. and Olsson, A.-M. (1998) Interaction between hemicelluloses, lignin, and cellulose: 
390

391

392

393

394

395

396

397

398

399

400

401

402

403

404

405

406

407

408

409

410

structure-property relationship, J. Pulp and Paper sci., 24; 99-103.

Samarasinghe, S., Loferski, J.R., Holzer, S.M. (1994) Creep modeling of wood using time-temperature superposition, Wood and Fiber, 26; 122-130.

Simon, P.P. and Ploehin H.J. (1999) Investigating time-temperature superpositioning in crosslinked polymers using the tube-junction model, J. Polym. Sci., 37; 127-142.

Stephen, S., Kelley, S., Timothy, G.P., Glasser, W.G.: Relaxation behaviour of the amorphous components of wood, J Mater. Sci., 617-624 (1987).

Tajvidi, M., Falk, R.H., Hermanson, J.C. (2005) Time-temperature superposition principle applied to a kenaf-fiber/high-density polyethulene composite, J. Appl. Polym. Sci., 97; 1995-2004.

Tsubaki, T., and Nakano, T. (2010). Creep behavior of bamboo under various desorption conditions, Holzforschung, 64; 489-493.

Tschoegl, N.W., Knauss, W.G., Emri, I. (2002) The effect of temperature and pressure on the mechanical properties of thermo- and/or piezorheologically simple polymeric materials in thermodynamic equilibrium - a critical review, Mech. Time-depend. Mater., 6; 53-99.

Voight, W. (1889) Ueber die Beziehung zwischen den beiden Elastizitlttskonstanten isotroper KSrper, Wied. Ann. 38; 573.

Williams, M.L., Landel, R.F., Ferry, J.D. (1955) The temperature dependence of relaxation mechanisms in amorphous polymers and other glass-forming liquids, J. Am. Chem. Soc., 77; 3701-3707.

Yoshitomi, T., Nagamatsu, K., Kosjiyama, K. (1958) On the stress relaxation of Nylone 6, J. Polym. Sci., 27; 335-347. 


\section{Caption}

413

414

415 Fig. 1. Shift of the relaxation modulus along a logarithmic time axis and correction along a logarithmic

416 vertical axis for the application of the time-temperature superposition principle (TTSP).

417

418

419

420

421

422

423

424

425

426

427

428

429

430

431

432

433

434

435

436

437

438

439

440

441

442

443

444

445 


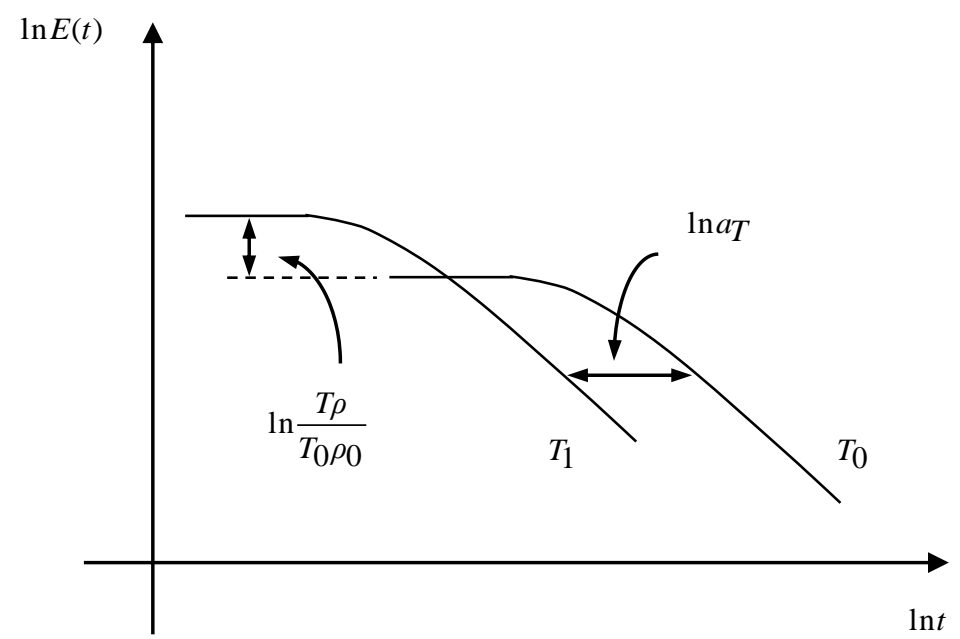

465

466

467 Fig. 1. Shift of the relaxation modulus along a logarithmic time axis and correction along a logarithmic 468 vertical axis for the application of the time-temperature superposition principle (TTSP). 